\title{
A Facile Synthesis of 2,4-Disubstituted Thiazoles Using $\mathrm{MnO}_{2}$
}

\author{
Yan-Bo Yu, Hong-Liang Chen, Li-Yi Wang, Xin-Zheng Chen and Bin Fu * \\ Department of Applied Chemistry, China Agricultural University, 100193, Beijing, China; \\ E-Mails: yuyanbo0902@163.com (Y.-B.Y.); chenhongliang1016@yahoo.cn (H.-L.C.); \\ Ritaxiaoyi@163.com (L.-Y.W.); xinzheng1943@126.com (X.-Z.C.)
}

* Author to whom correspondence should be addressed; E-Mail: fubinchem@cau.edu.cn.

Received: 21 October 2009; in revised form: 10 November 2009 / Accepted: 11 November 2009 / Published: 26 November 2009

\begin{abstract}
Structurally diverse thiazoles with electron-donating and electron-withdrawing groups were conveniently synthesized through manganese dioxide $\left(\mathrm{MnO}_{2}\right)$ oxidation of the corresponding thiazolines. The effect of substitution at the 2- and 4-positions was investigated. The desired thiazoles with aryl or vinyl substitutions at the 2- or 4-position can be obtained in good to excellent yields.
\end{abstract}

Keywords: thiazole; thiazoline; manganese dioxide; oxidation

\section{Introduction}

The thiazole ring is an interesting building block in a variety of natural products and bioactive compounds useful as pharmaceuticals or agrochemical agents [1-5], and to date many methods have been developed for the construction of thiazole ring systems. One classical and widely used method is the condensation of $\alpha$-haloketones with thioamide derivatives, which is known as the Hantzsch reaction [6-8]. Another efficient method is the introduction of substitutions onto a thiazole core structure through Stille coupling [9], which involves the use of organostannane intermediates. In recent years, a new and frequently encountered method for thiazole synthesis is the conversion of thiazoline derivatives through the use of dehydrogenating reagents such as sulfur [10], $\mathrm{KMnO}_{4}$ [11], $\mathrm{Cu}(\mathrm{I}) / \mathrm{Cu}(\mathrm{II}) /$ peroxide oxidation [12], $\mathrm{MnO}_{2}$ [13-16], NaH/DBU [17], and so on. Among these dehydrogenating reagents, activated $\mathrm{MnO}_{2}$ is a very simple and convenient reagent for the synthesis of thiazoles from thiazolines. However, all cases of $\mathrm{MnO}_{2}$ oxidation of thiazolines reported in the literature are restricted to thiazoles bearing electron-withdrawing substituents such as carboxylates, 
and to the best of our knowledge, no report involving the use of $\mathrm{MnO}_{2}$ for the synthesis of thiazoles without carboxylate substitution has appeared. To investigate the generality and scope of this method as a continuation of our research interest in thiazoline chemistry [18-20], we would like to report the synthesis of 2,4-disubstituted thiazoles with electron-donating and electron-withdrawing groups from the corresponding thiazolines via activated $\mathrm{MnO}_{2}$ oxidation.

\section{Results and Discussion}

The starting thiazolines 2 were easily prepared in one-pot reactions from the corresponding carboxylic acids 3 or their derivatives [19,20], and commercially available amino alcohols which provide $\mathrm{R}^{2}$ in the product (Scheme 1).

Scheme 1. The synthesis of 2,4-disubstituted thiazoles.

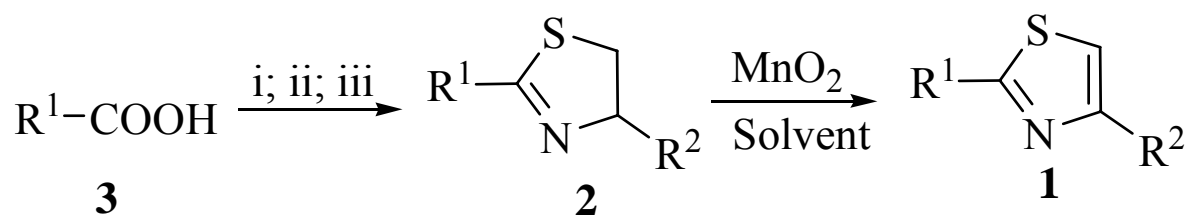

i) $\mathrm{SOCl}_{2}$; ii) amino alcohol, $\mathrm{Et}_{3} \mathrm{~N}$; iii) $\mathrm{P}_{2} \mathrm{~S}_{5}, \mathrm{Et}_{3} \mathrm{~N}$

With all kinds of thiazoline derivatives in hand, we first set out to optimize the reaction conditions. The suspension of thiazoline 2a and excess activated $\mathrm{MnO}_{2}$ (10 equiv.) in dichloromethane (DCM) was stirred for $24 \mathrm{~h}$ at room temperature $[15,16]$. No desired product was yielded even the reaction temperature was elevated to the boiling point for 48 hours. Through extensive screening of solvents, we observed that the reaction proceeded well under reflux in solvents with different polarity but similar boiling points. The results indicated the strong correlation between the yield and the reaction temperature. In DCE, $\mathrm{CH}_{3} \mathrm{CN}$, or benzene, full conversion and up to $95 \%$ isolated yields can be achieved within 12 hours. In the case of toluene, the starting material disappeared within $6 \mathrm{~h}$ and the thiazole product was afforded in $80 \%$ yield. Lowering the ratio of oxidant to thiazoline led to the significant decrease of the reaction rate. With the optimized condition in hand (DCE as solvent, 1:10 molar ratio of thiazoline to $\mathrm{MnO}_{2}$ ), thiazoles with different substitutions at the 2- and 4-positions were synthesized (Table 1). In most cases, the reaction proceeded well under reflux (entries 6-16). When one of 2- and 4-position of thiazoline is an aryl or vinyl group, the thiazole products are produced in good to excellent yields (entries 6, 7, 9, 10, 11, 14 and 16). When both the 2- and 4-position of thiazoline are aryl groups, the yields were improved to $95 \%-99 \%$ (entries 8,12 and 13), which can be ascribed to the stronger conjugation effect between aryl groups and thiazoles. In contrast, when both 2- and 4-positions of thiazoline are alkyl groups, none of the desired thiazole products was obtained (entry 17). The scope of this method was further exploited to the preparation of bis-thiazoles (Scheme 2). The desired products were also obtained in high yield from corresponding bis-thiazolines, as illustrated in Table 2 (entries 1-4). 
Table 1. The conversion of thiazolines to thiazoles by $\mathrm{MnO}_{2}$ oxidation ${ }^{\mathrm{a}}$.

\begin{tabular}{ccccccc}
\hline Entry & Compd. & $\mathbf{R}^{\mathbf{1}}$ & $\mathbf{R}^{\mathbf{2}}$ & Solvent & Time(h) & Yield(\%) \\
\hline 1 & $\mathbf{1 a}$ & $\mathrm{Ph}$ & $\mathrm{Me}$ & $\mathrm{DCM}$ & 48 & - \\
2 & $\mathbf{1 a}$ & $\mathrm{Ph}$ & $\mathrm{Me}$ & $\mathrm{DCE}$ & 12 & 95 \\
3 & $\mathbf{1 a}$ & $\mathrm{Ph}$ & $\mathrm{Me}$ & Benzene & 12 & 90 \\
4 & $\mathbf{1 a}$ & $\mathrm{Ph}$ & $\mathrm{Me}$ & $\mathrm{CH}_{3} \mathrm{CN}$ & 12 & 90 \\
5 & $\mathbf{1 a}$ & $\mathrm{Ph}$ & $\mathrm{Me}$ & Toluene & 6 & 80 \\
6 & $\mathbf{1 b}$ & $\mathrm{Ph}$ & $i$-Pr & $\mathrm{DCE}$ & 12 & 90 \\
7 & $\mathbf{1 c}$ & $\mathrm{Ph}$ & $i$ - $\mathrm{Bu}$ & $\mathrm{DCE}$ & 12 & 90 \\
8 & $\mathbf{1 d}$ & $\mathrm{Ph}$ & $\mathrm{Ph}$ & $\mathrm{DCE}$ & 12 & 99 \\
9 & $\mathbf{1 e}$ & $2-\mathrm{Py}$ & $\mathrm{Me}$ & $\mathrm{DCE}$ & 12 & 90 \\
10 & $\mathbf{1 f}$ & $2-\mathrm{Py}$ & $i$-Pr & $\mathrm{DCE}$ & 12 & 77 \\
11 & $\mathbf{1 g}$ & $2-\mathrm{Furyl}$ & $\mathrm{Bn}$ & $\mathrm{DCE}$ & 12 & 70 \\
12 & $\mathbf{1 h}$ & $2-\mathrm{Furyl}$ & $\mathrm{Ph}$ & $\mathrm{DCE}$ & 12 & 95 \\
13 & $\mathbf{1 i}$ & $2-$ thienyl & $\mathrm{Ph}$ & $\mathrm{DCE}$ & 12 & 95 \\
14 & $\mathbf{1 j}$ & $\mathrm{PhCH}=\mathrm{CH}-$ & $i$-Pr & $\mathrm{DCE}$ & 12 & 80 \\
15 & $\mathbf{1 k}$ & $\mathrm{PhCH}=\mathrm{CH}-$ & $\mathrm{Ph}$ & $\mathrm{DCE}$ & 12 & 95 \\
16 & $\mathbf{1 l}$ & $\mathrm{Me}$ & $\mathrm{Ph}$ & $\mathrm{DCE}$ & 12 & 76 \\
17 & $\mathbf{1 m}$ & $\mathrm{Me}$ & $i$-Pr & $\mathrm{DCE}$ & 24 & - \\
\hline
\end{tabular}

${ }^{\mathrm{a}}$ The reactions were run under reflux in different solvents.

Scheme 2. The synthesis of 2,4-disubstituted bis-thiazoles.<smiles>[R]C1=NC([R])CS1</smiles>

45

Table 2. The conversion of bis-thiazolines to bis-thiazoles by $\mathrm{MnO}_{2}$ oxidation ${ }^{\mathrm{a}}$.

\begin{tabular}{|c|c|c|c|c|c|}
\hline Entry & Compd. & $\mathbf{R}^{1}$ & $\mathbf{R}^{2}$ & Reaction time & Yield \\
\hline 1 & $5 a$ & & $i-\operatorname{Pr}$ & 12 & 80 \\
\hline 2 & $5 b$ & & $\mathrm{Me}$ & 12 & 85 \\
\hline 3 & $5 c$ & & $i-\operatorname{Pr}$ & 6 & 80 \\
\hline 4 & 5d & & $\mathrm{Ph}$ & 8 & 70 \\
\hline
\end{tabular}

${ }^{\mathrm{a}}$ The reactions were run under reflux in DCE. 


\section{Conclusions}

In conclusion, we have demonstrated that thiazoles bearing different electron-donating and electron-withdrawing groups can be conveniently synthesized from the corresponding thiazolines using activated $\mathrm{MnO}_{2}$ in dichloroethane. The critical effects of the reaction temperature and the substitutions on the thiazoline ring were investigated. The scope of this method was further extended to the preparation of 2,4-disubstituted thiazoles with diverse groups.

\section{Experimental}

NMR spectra were recorded on a Bruker Avance DPX300 spectrometer with tetramethylsilane as internal standard and $\mathrm{CDCl}_{3}$ as solvent. Infrared spectra were obtained on a Nicolet AVATAR 330 FT-IR spectrometer. Elemental analyses were carried out on an Elementar Vario EL instrument. Melting points were measured on an XT-4 melting point apparatus and were uncorrected. Solvents were purified and dried following standard procedures.

\subsection{Synthesis of Thiazolines}

All thiazolines were prepared according to the literature [19,20].

\subsection{Typical Procedure for Oxidation of Thiazolines to Thiazoles}

To a solution of 4-methyl-2-phenylthiazoline $(177 \mathrm{mg}, 1 \mathrm{mmol})$ in 1,2-dichloroethane $(10 \mathrm{~mL})$ was added activated $\mathrm{MnO}_{2}(860 \mathrm{mg}, 10 \mathrm{mmol})$. The mixture was then refluxed for $12 \mathrm{~h}$ under a nitrogen atmosphere. After filtration, the mixture was evaporated in vacuo. The residue was chromatographed on silica gel (ethyl Acetate-hexane, 10:1) to give $176 \mathrm{mg}$ (95\% yield) of 4-methyl-2-phenylthiazole (1a) [21] as a colorless oil; ${ }^{1} \mathrm{H}-\mathrm{NMR}$ : $\delta$ 7.94-7.91(m, 2H), 7.43-7.39 (m, 3H), 6.85 (t, $J=0.96 \mathrm{~Hz}$, 1H), $2.50(\mathrm{~d}, J=0.96 \mathrm{~Hz}, 3 \mathrm{H})$; ${ }^{13} \mathrm{C}-\mathrm{NMR}: \delta 167.44,153.71,133.72,129.65,128.75,126.34,113.30,17.14$.

\subsection{Spectral Data of Other Thiazole Compounds}

$1 \boldsymbol{b}$ [11]: ${ }^{1} \mathrm{H}-\mathrm{NMR}: \delta$ 7.96-7.91(m, 2H, ArH), 7.44-7.37 (m, 3H, ArH), 6.86 (s, 1H), 3.21-3.11 (m, 1H), 1.35 (d, $J=6.90 \mathrm{~Hz}, 6 \mathrm{H}) ;{ }^{13} \mathrm{C}-\mathrm{NMR}: \delta 167.29,164.87,134.08,129.63,128.79,126.52,110.88$, $31.05,22.40$.

1c: colorless oil; IR $\left(\mathrm{KBr}, \mathrm{cm}^{-1}\right)$ : 3063, 2955, 2928, 1516, 1461, 1244, 763; ${ }^{1} \mathrm{H}-\mathrm{NMR}: \delta$ 7.95-7.91 (m, 2H, ArH), 7.49-7.35 (m, 3H, ArH), 6.85 (d, $J=0.63 \mathrm{~Hz}, 1 \mathrm{H}), 2.67$ (dd, $J=9.0,0.75 \mathrm{~Hz}, 2 \mathrm{H})$, $2.16-2.06(\mathrm{~m}, 1 \mathrm{H}), 0.97(\mathrm{~d}, J=6.60 \mathrm{~Hz}, 6 \mathrm{H}) ;{ }^{13} \mathrm{C}-\mathrm{NMR}: \delta 167.16,157.77,133.93,129.57,128.74$, 128.43, 113.45, 40.78, 28.38, 22.35; Anal. Calcd. for $\mathrm{C}_{13} \mathrm{H}_{15} \mathrm{NS}$ (217.34): C 71.84, H 6.96, N 6.44. Found: C 71.96, H 6.85, N 6.23 .

1d [22]: white solid, mp: $90.5{ }^{\circ} \mathrm{C}-92.0{ }^{\circ} \mathrm{C}$ (lit. [22] 91.0-92.0 ${ }^{\circ} \mathrm{C}$ ); ${ }^{1} \mathrm{H}-\mathrm{NMR}: \delta 8.05-7.98(\mathrm{~m}, 4 \mathrm{H}$ ), 7.47-7.42 (m, 6H), 7.41-7.34 (m, 1H); ${ }^{13} \mathrm{C}-\mathrm{NMR}: \delta 167.74,156.21,134.48,133.72,129.53,128.83$, $128.65,128.08,126.54,126.34,112.54$. 
1e [23]: white solid, mp: 85.0-86.0 ${ }^{\circ} \mathrm{C}$ (lit. [23] 84.0-84.5 $\left.{ }^{\circ} \mathrm{C}\right) ;{ }^{1} \mathrm{H}-\mathrm{NMR}: \delta 8.60-8.58(\mathrm{~m}, 1 \mathrm{H})$, 8.18-8.14 (m, 1H), 7.79-7.73 (m, 1H), 7.30-7.26 (m, 1H), 6.99 (d, $J=0.84 \mathrm{~Hz}, 1 \mathrm{H}), 2.52(\mathrm{~d}, J=0.84$ $\mathrm{Hz}, 3 \mathrm{H}) ;{ }^{13} \mathrm{C}-\mathrm{NMR}: \delta 167.92,153.81,151.12,149.06,136.53,123.87,119.17,115.84,16.96$.

1f: colorless oil; IR $\left(\mathrm{KBr}, \mathrm{cm}^{-1}\right): 3060,2920,1738,1365,1217 ;{ }^{1} \mathrm{H}-\mathrm{NMR}: \delta 8.60-8.58(\mathrm{~m}, 1 \mathrm{H})$, 8.21-8.18 (m, 1H), 7.79-7.73 (m, 1H), 7.29-7.25 (m, 1H), 6.98 (d, J=0.84 Hz, 1H), 3.19-3.14 (m, 1H), $1.36(\mathrm{~d}, J=6.90 \mathrm{~Hz}, 6 \mathrm{H}) ;{ }^{13} \mathrm{C}-\mathrm{NMR}: \delta 167.96,165.07,151.61,149.26,136.71,124.01,119.57$, 113.45, 30.96, 22.31; Anal. Calcd. for $\mathrm{C}_{11} \mathrm{H}_{12} \mathrm{~N}_{2} \mathrm{~S}$ (204.30): C 64.67, H 5.92, N 13.71. Found: C 64.88, H 5.91, N 13.45 .

1g: colorless oil; IR (KBr, $\left.\mathrm{cm}^{-1}\right): 3120,1569,1495,1473,1299,1133,810,769 ;{ }^{1} \mathrm{H}-\mathrm{NMR}: \delta 7.49$ $(\mathrm{t}, J=1.20 \mathrm{~Hz}, 1 \mathrm{H}), 7.35-7.22(\mathrm{~m}, 5 \mathrm{H}, \mathrm{ArH}), 6.97(\mathrm{dd}, J=2.1,0.6 \mathrm{~Hz}, 1 \mathrm{H}), 6.69(\mathrm{~s}, 1 \mathrm{H}), 6.51$ $(\mathrm{dd}, J=4.80,3.33 \mathrm{~Hz}, 1 \mathrm{H}), 4.17$ (s, 2H); ${ }^{13} \mathrm{C}-\mathrm{NMR}: \delta 157.81,151.51,149.04,143.41,138.89,129.08$, 128.54, 126.48, 113.59, 112.08, 108.79, 37.91; Anal. Calcd. for $\mathrm{C}_{14} \mathrm{H}_{11} \mathrm{NOS}$ (241.32): C 69.68, H 4.59, N 5.80. Found: C 69.75, H 4.85, N 5.93.

1h: white solid, mp: $72.3-72.9^{\circ} \mathrm{C}$; IR $\left(\mathrm{KBr}, \mathrm{cm}^{-1}\right)$ : 3060, 2970, 1738, 1452, 1217, 1015, 750; ${ }^{1} \mathrm{H}-\mathrm{NMR}: 7.96(\mathrm{~d}, J=1.32 \mathrm{~Hz}, 1 \mathrm{H}), 7.94(\mathrm{~s}, 1 \mathrm{H}), 7.53$ (d, $\left.J=1.14 \mathrm{~Hz}, 1 \mathrm{H}\right), 7.46-7.32$ (m, 4H, ArH), $7.08(\mathrm{~d}, J=3.45 \mathrm{~Hz}, 1 \mathrm{H}), 6.55(\mathrm{dd}, J=3.30,1.80 \mathrm{~Hz}, \mathrm{ArH}) ;{ }^{13} \mathrm{C}-\mathrm{NMR}: 157.79,156.26,149.09$, 143.48, 134.19, 128.66, 128.20, 126.46, 112.13, 111.83, 108.98; Anal. Calcd. for $\mathrm{C}_{13} \mathrm{H}_{9} \mathrm{NOS}(227.89)$ : C: 68.52, H: 3.98, N: 6.15. Found: C 68.66, H 4.05, N 6.13.

1i [24]: colorless oil; ${ }^{1} \mathrm{H}-\mathrm{NMR}: \delta 7.96-7.93(\mathrm{~m}, 2 \mathrm{H}), 7.52(\mathrm{dd}, J=3.60,1.14 \mathrm{~Hz}, 1 \mathrm{H}), 7.44-7.32(\mathrm{~m}, 5 \mathrm{H})$, $7.05(\mathrm{dd}, J=5.40,3.60 \mathrm{~Hz}, 1 \mathrm{H}) ;{ }^{13} \mathrm{C}-\mathrm{NMR}: \delta 161.15,155.56,137.30,133.95,128.50,127.99,127.60$, $127.45,126.38,126.26,111.74$.

1j: colorless oil; IR (KBr, cm $\left.{ }^{-1}\right): 3034,1738,1476,1365,1217 ;{ }^{1} \mathrm{H}-\mathrm{NMR}: \delta 7.52-7.48$ (m, 2H, ArH), 7.38-7.24 (m, 5H, ArH), $6.78(\mathrm{~s}, 1 \mathrm{H}),, 3.16-3.06(\mathrm{~m}, 1 \mathrm{H}), 1.33(\mathrm{~d}, J=6.90 \mathrm{~Hz}, 6 \mathrm{H})$; ${ }^{13} \mathrm{C}-\mathrm{NMR}$ : $\delta$ 166.20, 164.50, 135.85, 133.72, 128.70, 128.57, 126.89, 121.88, 110.26, 30.89, 22.29; Anal. Calcd. for $\mathrm{C}_{14} \mathrm{H}_{15} \mathrm{NS}$ (229.35): C 73.32, H 6.59, N 6.11. Found: C 73.55, H 6.72, N 6.33.

$1 \boldsymbol{k}$ [25]: colorless oil; ${ }^{1} \mathrm{H}-\mathrm{NMR}: \delta$ 7.95-7.92 (m, 2H), 7.58-7.55 (m, 2H), 7.47-7.32 (m, 9H); ${ }^{13} \mathrm{C}-\mathrm{NMR}: \delta 166.76,156.26,135.82,134.52,134.42,128.88,128.75,128.70,128.20,127.12,126.44$, 121.68, 112.09 .

11 [26]: white solid, mp: 64.0-65.5 ${ }^{\circ} \mathrm{C}$ (lit. [26] 64 ${ }^{\circ} \mathrm{C}$ ); ${ }^{1} \mathrm{H}-\mathrm{NMR}: \delta$ 7.89-7.85 (m, 2H), 7.44-7.38 (m, 2H), 7.34-7.28 (m, 2H), 2.77 (s, 3H); ${ }^{13} \mathrm{C}-\mathrm{NMR}: \delta$ 165.80, 155.22, 134.59, 129.01, 128.69, 127.95, 126.54, 126.34, 112.19, 19.31 .

5a: colorless oil; IR $\left(\mathrm{KBr}, \mathrm{cm}^{-1}\right): 2961,1569,1509,1429,1270,742 ;{ }^{1} \mathrm{H}-\mathrm{NMR}: \delta 8.45(\mathrm{t}, J=1.75 \mathrm{~Hz}$, 1H), $7.98(\mathrm{dd}, J=7.80,1.50 \mathrm{~Hz}, 2 \mathrm{H}), 7.47$ (t, $J=7.80 \mathrm{~Hz}, 1 \mathrm{H}), 6.90$ (d, $J=0.72 \mathrm{~Hz}, 1 \mathrm{H}), 3.23-3.13$ $(\mathrm{m}, 2 \mathrm{H}), 1.37(\mathrm{~d}, J=6.90 \mathrm{~Hz}, 12 \mathrm{H}) ;{ }^{13} \mathrm{C}-\mathrm{NMR}: \delta 166.38,164.89,134.65,129.23,127.53,124.37$, 111.22, 30.98, 22.32; Anal. Calcd. for $\mathrm{C}_{18} \mathrm{H}_{20} \mathrm{~N}_{2} \mathrm{~S}_{2}$ (328.51): C 65.81, H 6.14, N 8.53. Found: C 65.95, H $6.25, \mathrm{~N} 8.44$. 
$5 \boldsymbol{b}$ [27]: white solid, mp: $126-126.5^{\circ} \mathrm{C}$; ${ }^{1} \mathrm{H}-\mathrm{NMR}: \delta 8.14(\mathrm{~d}, J=7.80 \mathrm{~Hz}, 2 \mathrm{H}), 7.86(\mathrm{t}, J=7.80 \mathrm{~Hz}, 1 \mathrm{H})$, $7.02(\mathrm{~d}, J=0.90 \mathrm{~Hz}, 2 \mathrm{H}), 2.53(\mathrm{~d}, J=0.85 \mathrm{~Hz}, 6 \mathrm{H}) ;{ }^{13} \mathrm{C}-\mathrm{NMR}: \delta 165.80,155.22,134.59,128.69$, $127.95,126.34,112.19,19.31$.

5c: white solid, $\mathrm{mp}: 61.5-62.0^{\circ} \mathrm{C}$; IR $\left(\mathrm{KBr}, \mathrm{cm}^{-1}\right): 3068,2926,1564,1510,1498,1011,669$; ${ }^{1} \mathrm{H}-\mathrm{NMR}: \delta 8.17(\mathrm{~d}, J=7.80 \mathrm{~Hz}, 2 \mathrm{H}), 7.85(\mathrm{t}, J=7.80 \mathrm{~Hz}, 1 \mathrm{H}), 7.01(\mathrm{~d}, J=0.66 \mathrm{~Hz}, 2 \mathrm{H}), 3.21-3.12$ $(\mathrm{m}, 2 \mathrm{H}), 1.37(\mathrm{~d}, J=6.90 \mathrm{~Hz}, 12 \mathrm{H}) ;{ }^{13} \mathrm{C}-\mathrm{NMR}: \delta 167.68,165.28,151.28,137.80,119.86,113.93$, 31.10, 22.44; Anal. Calcd. for $\mathrm{C}_{17} \mathrm{H}_{19} \mathrm{~N}_{3} \mathrm{~S}_{2}$ (329.50): C: 61.97, H: 5.81, N: 12.75. Found: C: 61.99, H: $5.85, \mathrm{~N}: 12.90$.

5d: colorless oil; IR $\left(\mathrm{KBr}, \mathrm{cm}^{-1}\right)$ : 2920, 1569, 1485, 1270, 1174, 1072, 731; ${ }^{1} \mathrm{H}-\mathrm{NMR}: \delta 7.98(\mathrm{~d}$, $J=1.38 \mathrm{~Hz}, 4 \mathrm{H}), 7.45-7.30(\mathrm{~m}, 8 \mathrm{H}), 3.11(\mathrm{t}, J=7.74 \mathrm{~Hz}, 4 \mathrm{H}), 2.25-2.20(\mathrm{~m}, 2 \mathrm{H}) ;{ }^{13} \mathrm{C}-\mathrm{NMR}: \delta 174.92$, 155.01, 134.67, 128.69, 127.99, 126.42, 113.29, 51.32, 36.64, 16.61; Anal. Calcd. for $\mathrm{C}_{22} \mathrm{H}_{18} \mathrm{~N}_{2} \mathrm{~S}_{2}$ (374.54): C 70.55, H 4.84, N 7.48. Found: C 70.69, H 4.85, N 7.62.

\section{Acknowledgements}

This work was financially supported by the Ministry of Science and Technology of China (No. 2006BAE01A01) and the Innovation Programme for National Undergraduated Students.

\section{References and Notes}

1. Rivkin, A.; Cho, Y.S.; Gabarda, A.E.; Yoshimura, F.; Danishefsky, S.J. Application of RingClosing Metathesis Reactions in the Synthesis of Epothilones. J. Nat. Prod. 2004, 67, 139-143.

2. Ganesh, T.; Schilling, J.K.; Palakodety, R.K.; Ravindra, R.; Shanker, N.; Bane, S.; Kingston, D.G.I. Synthesis and biological evaluation of fluorescently labeled epothilone analogs for tubulin binding studies. Tetrahedron 2003, 59, 9979-9984.

3. Plazzi, P.V.; Bordi, F.; Silva, C.; Morini, G.; Caretta, A.; Barocelli, E.; Vitali, T. Heteroarylaminoethyl and heteroaryithioethyl imidazoles. Synthesis and H3-receptor affinity. Eur. J. Med. Chem. 1995, 30, 881-889.

4. Bai, N.; Sha, Y.W.; Meng, G. Efficient and Eco-Friendly Preparation of 4-Methyl-5-formylthiazole. Molecules 2008, 13, 943-947.

5. Hu, D.J.; Liu, S.F.; Huang, T.H.; Tu, H.Y.; Zhang, A.D. Synthesis and herbicidal activities of novel 4-(4-(5-methyl-3-arylisoxazol-4-yl)thiazol-2-yl)piperidyl carboxamides and thio-carboxamides. Molecules 2009, 14, 1288-1303.

6. Aitken, K.M.; Aitken, R.A. Synthesis of 2,4-diacetylthiazole and 2,5-diacetylthiazole. Tetrahedron 2008, 64, 4384-4386.

7. Potewar, T.M.; Ingale, S.A.; Srinivasan, K.V. Efficient synthesis of 2, 4-disubstituted thiazoles using ionic liquid under ambient conditions: A practical approach towards the synthesis of Fanetizole. Tetrahedron 2007, 63, 11066-11069.

8. Narender, M.; Reddy, M.S.; Sridhar. R.; Nageswar, Y.V.D.; Rao, K.R. Aqueous phase synthesis of thiazoles and aminothiazoles in the presence of $\beta$-cyclodextrin. Tetrahedron Lett. 2005, 46, 5953-5955. 
9. Hämmerle, J.; Spina, M., Schnürch, M.; Mihovilovic, M.D.; Stanetty, P. A Comparative Study on Stille Cross-Coupling Reactions of 2-Phenylthiazoles and 2-Phenyloxazoles. Synthesis 2008, 19, 3099-3107.

10. Friedrich, A.; Max, T.; Karl, G. Concomitant reaction of elementary sulfur and gaseous ammonia on ketones. XXIX. Syntheses and properties of 2-aryl-3-thiazolines and 2-arylthiazoles. Justus Liebigs Ann. Chem. 1961, 639, 133-146.

11. Aitken, R.A.; Armstrong, D.P.; Galt, R.H.B.; Mesher, S.T.E. Synthesis and oxidation of chiral 2thiazolines (4,5-dihydro-1,3-thiazoles). J. Chem. Soc. Perkin Trans. 1997, 1, 935-943.

12. Meyers, A.I.; Tavares, F.X. Oxidation of oxazolines and thiazolines to oxazoles and thiazoles application of the Kharasch-Sosnovsky reaction. J. Org. Chem. 1996, 61, 8207-8215.

13. Fernandez, X.; Duňach, E. Asymmetric synthesis of 2-alkyl-3-thiazoline carboxylates: stereochemistry of the $\mathrm{MnO}_{2}$-mediated oxidation of cis- and trans-2-alkyl-thiazolidine-(4R)carboxylates. Tetrahedron Asymmetry 2001, 12, 1279-1286.

14. Fernandez, X.; Fellous, R.; Lizzani-Cuvelier, L.; Loiseau, M.; Duňach, E. Chemo- and regioselective synthesis of alkyl-3-thiazoline carboxylates. Tetrahedron Lett. 2001, 42, 1519-1521.

15. You, S.L.; Kelly, J.W. The total synthesis of bistratamides F-I. Tetrahedron 2005, 61, 241-249.

16. You, S.L.; Kelly, J.W. Highly efficient biomimetic total synthesis and structural verification of Bistratamides E and J from Lissoclinum bistratum. Chem. Eur. J. 2004, 10, 71-75.

17. Mislin, G.L.; Burger, A.; Abdallah, M. A. Synthesis of new thiazole analogues of pyochelin, a siderophore of Pseudomonas aeruginosa and Burkholderia cepacia. A new conversion of thiazolines into thiazoles. Tetrahedron 2004, 60, 12139-12145.

18. Cheng, X.M.; Zheng, Z.B.; Li, N.; Qin, Z.H.; Fu, B.; Wang, N.D. Synthesis of novel C3 symmetric tris(thiazoline) ligands and their application in the allylic oxidation reaction. Tetrahedron Asymmetry 2008, 19, 2159-2163.

19. Lu, X.H.; Qi, Q.Q.; Xiao, Y.M.; Li, N.; Fu, B. A convenient one-pot synthesis of arene-centered tris(thiazoline) compounds. Heterocycles 2009, 78, 1031-1039.

20. Liu, L.; Zheng, Z.B.; Qin, Z.H.; Fu, B. Yuan, H.Z. Synthesis and biological activity of 2-indolyl oxazoline and thiazoline derivatives. Chin. J. Org. Chem. 2008, 28, 1841-1845.

21. Taku, A.; Takuya, A.; Takahide, F.; Yutaka, I.; Fumitoshi, K.; Naoto, C. Ruthenium- and Rhodium-Catalyzed Direct Carbonylation of the Ortho C-H Bond in the Benzene Ring of NArylpyrazoles. J. Org. Chem. 2004, 69, 4433-4440.

22. Yoshihide, I.; Hideo, T. Facile preparation of thiazoles from 1H-1-(1'-alkynyl)-5-methyl-1,2,3benziodoxathiole 3,3-dioxide with thioamides. Synlett 2008, 17, 2637-2641.

23. Knott, R.F.; Breckenridge, J.G. Analogs of 2,2'-bipyridyl with isoquinoline and thiazole rings. Part I. Can. J. Chem. 1954, 32, 512-521.

24. Singh, S. P.; Subhash, S. Synthesis and phototoxicity of some 2-(phenyl- or 2- or 3-thienyl)-4substituted thiazoles. Indian J. Chem. Sect. B: Org. Chem. Incl. Med. Chem. 1988, 27B, 941-943.

25. Takao, S.; Hideo, N.; Yoshinori, K.; Masafumi, S.; Hiroshi, Y.; Palladium-catalyzed reactions of terminal acetylenes and olefins with halo-1,3-azoles. Chem. Pharm. Bull. 1987, 35, 823-828. 
26. Makoto, U.; Hideo, T. Environmentally benign preparation of heteroaromatics from ketones or alcohols, with macroporous polystyrenesulfonic acid and (diacetoxyiodo)benzene, followed by thioamide, amidine, and 2-aminopyridine. Synthesis 2004, 16, 2673-2677.

27. Veli-Matti, M.; Paivi, L.; Ilkka, H.; Harri, T.; Cristina, M.; Jouko, K. Novel thiazole-containing complexing agents and luminescence of their europium(III) and terbium(III) chelates. Helv. Chim. Acta 1996, 79, 295-306.

Sample Availability: Samples of the compounds are available from the authors.

(C) 2009 by the authors; licensee Molecular Diversity Preservation International, Basel, Switzerland. This article is an open-access article distributed under the terms and conditions of the Creative Commons Attribution license (http://creativecommons.org/licenses/by/3.0/). 